\title{
HBsAg-Negative, Anti-HBc-Negative Patients Still Have a Risk of Hepatitis B Virus-Related Hepatitis after Autologous Stem Cell Transplantation for Multiple Myeloma or Malignant Lymphoma
}

\author{
Hyunsung Park, MD, MS ${ }^{1}$ \\ Do Young Kim, MD, PhD² \\ Soo-Jeong Kim, MD, MS ${ }^{1}$ \\ Haerim Chung, MD ${ }^{1}$ \\ Hyunsoo Cho, MD, MS' \\ Ji Eun Jang, MD ${ }^{1}$ \\ June-Won Cheong, MD, PhD' \\ Yoo Hong Min, MD, PhD' \\ Jae-Woo Song, MD, $P h D^{3}$ \\ Jin Seok Kim, MD, PhD'
}

\begin{abstract}
Purpose
Although hepatitis B surface antigen (HBsAg)-negative, hepatitis B core antibody (anti-HBc)negative patients are not considered to be at risk for hepatitis B virus (HBV)-related hepatitis, the actual risk remains to be elucidated. This study aimed to evaluate the risk of HBV-related hepatitis in HBsAg-negative, anti-HBc-negative patients receiving autologous stem cell transplantation (ASCT) for multiple myeloma (MM) or malignant lymphoma.
\end{abstract}

\section{Materials and Methods}

We retrospectively reviewed data from $271 \mathrm{HBsAg-negative} \mathrm{patients} \mathrm{(161} \mathrm{anti-HBc-negative}$ and 110 anti-HBc-positive at the time of ASCT) who received ASCT for MM or lymphoma. The risk of HBV-related hepatitis was analyzed according to the presence of anti-HBc. HBV serology results at the time of ASCT were compared with those at the time of diagnosis of MM or lymphoma.

\section{Results}

Three patients (two anti-HBc-negative MMs and one anti-HBc-positive MM) developed HBV-related hepatitis after ASCT. The rate of HBV-related hepatitis did not differ among patients with or without anti-HBc status $(p=0.843)$. HBV-related hepatitis more frequently occurred in MM patients than in lymphoma patients ( $p=0.041$ ). Overall, $9.1 \%$ of patients (16.7\% with MM and 5.4\% with lymphoma) who were HBsAg-negative and anti-HBc-positive at the time of diagnosis had lost anti-HBc positivity during chemotherapy prior to ASCT.

\section{Conclusion}

Our data suggest that HBsAg-negative, anti-HBc-negative patients at the time of ASCT for MM or lymphoma still might be at a risk for HBV-related hepatitis.

\section{Key words}

Hepatitis B core antibody, HBV-related hepatitis,

Stem cell transplantation, Lymphoma, Multiple myeloma
Correspondence: Jin Seok Kim, MD, PhD Division of Hematology, Department of Internal Medicine, Severance Hospital, Yonsei University College of Medicine, 50-1 Yonsei-ro, Seodaemun-gu, Seoul 03722, Korea Tel: 82-2-2228-1972

Fax: 82-2-393-6884

E-mail: hemakim@yuhs.ac

Received July 10, 2017

Accepted November 27, 2017

Published Online December 4, 2017

${ }^{*}$ Hyunsung Park and Do Young Kim contributed equally to this work.

* ${ }^{*}$ This study was presented in the form of a poster presentation at the 56 th annual meeting of the American Society of Hematology in San Francisco, CA on December 6-9, 2014. 


\section{Introduction}

Hepatitis B virus (HBV)-related hepatitis is a serious complication resulting from HBV reactivation after immunosuppressive treatment in patients with resolved HBV. Resolved HBV infections are usually defined as hepatitis B surface antigen (HBsAg)-negative and hepatitis B core antibody (anti-HBc)-positive status in individuals with low $(<200$ $\mathrm{IU} / \mathrm{mL}$ ) or undetectable levels of HBV-DNA [1-3]. Patients who are HBsAg-negative and anti-HBc-negative are often classified as HBV naïve [1] and are not considered to be at a risk of developing HBV-related hepatitis in clinical practice.

Autologous stem cell transplantation (ASCT) is an effective approach for the treatment of multiple myeloma (MM) and malignant lymphoma. However, MM and lymphoma are known to increase the risk of HBV reactivation [4-6]. Therefore, $\mathrm{HBV}$-related hepatitis following $\mathrm{HBV}$ reactivation is an important clinical concern in MM and lymphoma patients with resolved HBV during or after ASCT. To prevent HBVrelated hepatitis, all patients with $\mathrm{MM}$ or lymphoma should be screened for HBsAg and anti-HBc prior to ASCT $[3,7,8]$. $\mathrm{HBV}$ prophylaxis or $\mathrm{HBV}$ pre-emptive therapy according to HBV-DNA results are recommended in HBsAg-negative, anti-HBc-positive patients by the European Association for the Study of the Liver (EASL) Clinical Practice Guidelines, although the American Association for the Study of Liver Diseases (AASLD) Practice Guidelines do not recommend routine HBV prophylaxis for these patients [3,7]. In HBsAgnegative, anti-HBc-negative patients, however, no guidelines suggest that these patients should be evaluated for the risk of HBV-related hepatitis. Nevertheless, HBsAg-negative, anti-HBc-negative patients prior to ASCT might still be at risk of developing HBV-related hepatitis: For instance, although anti-HBc is generally known to persist throughout one's lifetime, impaired production of anti-HBc can occur during chemotherapy prior to ASCT in patients who are HBsAg-negative and anti-HBc-positive at the time of diagnosis of MM or lymphoma [9]. This may result in erroneously interpreting a patient's HBV infection status at the time of ASCT as HBV-naïve if there is no known previous information. However, to our knowledge, the risk of HBVrelated hepatitis in patients who are HBsAg-negative and anti-HBc-negative has not been reported, and tends to be underestimated by physicians. Hence, we conducted this retrospective study to clarify the risk of HBV-related hepatitis in patients who are $\mathrm{HBsAg}$-negative and anti-HBc-negative at the time of ASCT for MM or lymphoma.

\section{Materials and Methods}

\section{Patients and study design}

Two hundred eighty-eight patients who were diagnosed with MM or malignant lymphoma received ASCT at Severance Hospital, Seoul, Korea between November 2005 and April 2014. All patients were tested for serologic markers of HBV prior to ASCT as a baseline. Among them, HBsAg-negative patients were enrolled in this study retrospectively. Patients who were not tested for anti-HBc at baseline were excluded (Fig. 1). Patients with HBsAg-negativity were not tested for HBV-DNA levels and did not receive anti-HBV agents, because reimbursement guidelines set by the Korean government did not permit such testing and treatment in these patients. We then analyzed the rates of HBV-related hepatitis after ASCT according to baseline anti-HBc status. As reconstitution of normal humoral and cellular immunity after ASCT may require 1 year or more [10], we considered HBV-related hepatitis that occurred within and more than 1 year after ASCT as early and delayed events, respectively. Next, the results on serologic markers of $\mathrm{HBV}$ at baseline were compared to those at the time of diagnosis of MM or lymphoma. Additionally, we also assessed the potential impact of rituximab on changes in HBV serology in lymphoma patients in sub-analysis.

\section{Definitions of hepatitis, HBV-related hepatitis, and HBV-related death}

Hepatitis was defined as a threefold or greater increase in serum alanine transaminase (ALT) levels that exceeded the reference range (5.0-46.0 U/L) or an absolute increase in ALT to greater than $100 \mathrm{U} / \mathrm{L}$ [11]. HBV reactivation is usually defined as the appearance of HBV-DNA or a more than 10-fold increase in serum HBV-DNA than that at baseline $[1,2]$. As serum HBV-DNA was not tested regularly, unless positive conversion of HBsAg (HBsAg reversion) was noted, $\mathrm{HBV}$ reactivation could not be identified. Therefore, we defined hepatitis attributed to HBV (HBV-related hepatitis) as the presence of HBsAg reversion with no clinical or laboratory features of acute infection with acute hepatitis A or C, any other systemic infection, or drug-induced hepatitis. The definition of HBV-related death was defined as any death resulting from HBV-related hepatitis.

\section{Determination of hepatitis and HBV-related hepatitis after ASCT}

After ASCT, liver function tests were conducted every 2 weeks for 3 months and monthly thereafter for at least 2 


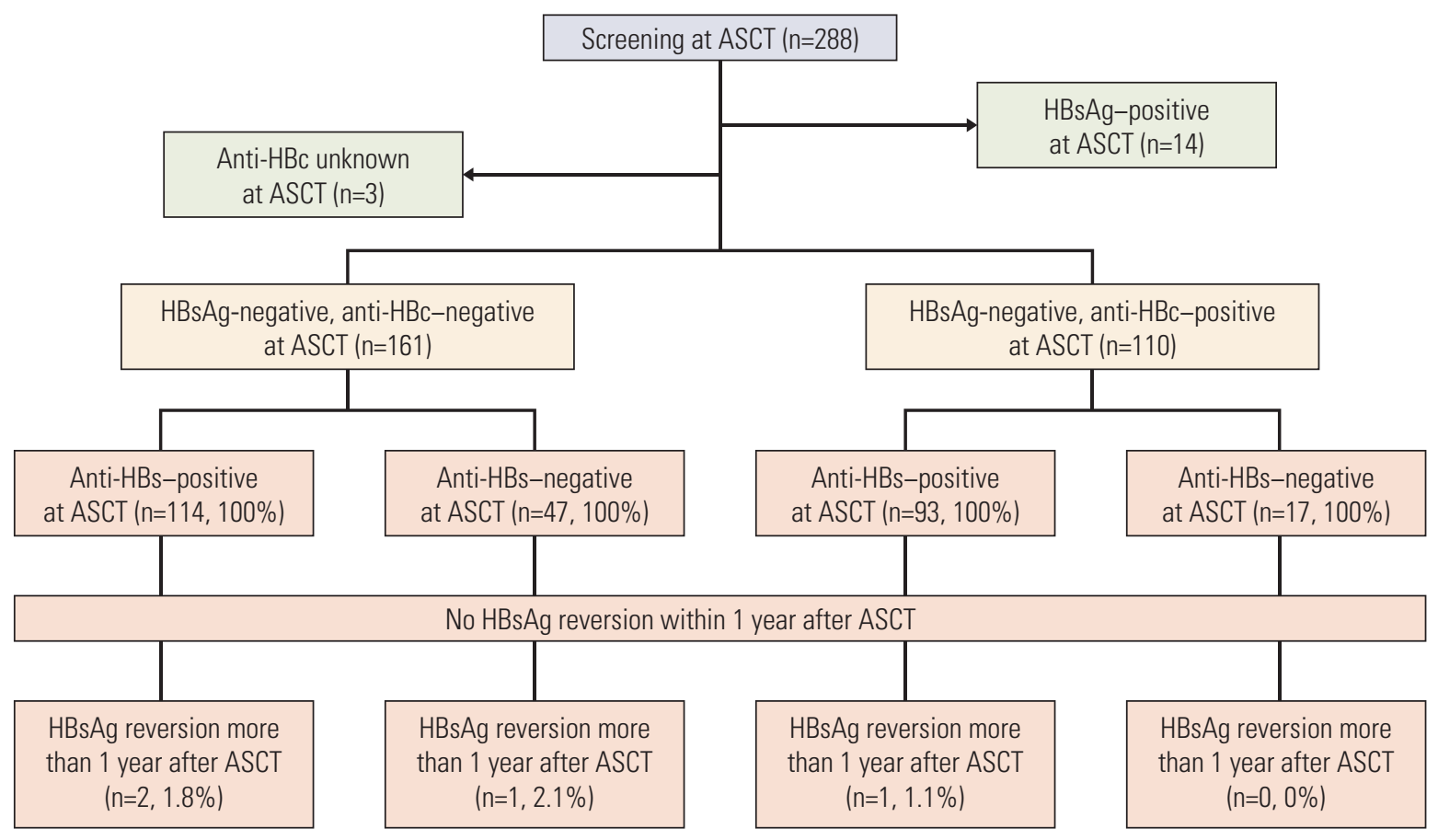

Fig. 1. The incidence of hepatitis and hepatitis B surface antigen (HBsAg) reversion in 271 HBsAg-negative patients within and more than 1 year after autologous stem cell transplantation without hepatitis B virus (HBV) prophylaxis. Anti-HBc, hepatitis B core antibody; Anti-HBs, hepatitis B surface antibody; ASCT, autologous stem cell transplantation.

years. HBV serologic markers were tested if HBV-related hepatitis was clinically suspected. Serum HBV-DNA levels were measured if HBsAg reversion was detected. Serum HBV-DNA levels were quantified with a real-time polymerase chain reaction assay on a Cobas TaqMan 48 analyzer (Roche Molecular Systems, Branchburg, NJ). The lower detection limit was 60 copies $/ \mathrm{mL}$.

\section{Statistical analysis}

Frequencies between groups were evaluated and compared using chi-square or Fisher exact tests. Mann-Whitney $\mathrm{U}$ tests were applied for continuous variables. A binary logistic regression test was applied for multivariate analysis. Two-tailed p-values less than 0.05 were considered significant. All statistical analyses were performed using SPSS software ver. 20.0 (IBM Corp., Armonk, NY).

\section{Ethical statement}

The present study conformed to the ethical guidelines of the World Medical Association Declaration of Helsinki and was approved by the Institutional Review Board of Severance Hospital (IRB approval number: 4-2014-0958). Informed consent was waived because the study was based on retrospective review of clinical data.

\section{Results}

\section{Patient characteristics}

Of the 288 patients who were initially screened, 274 were HBsAg-negative. Of these, three patients were not tested for anti-HBc at the time of ASCT and excluded from this study (Fig. 1). The baseline characteristics of the remaining 271 patients are summarized in Table 1. Information on previous vaccinations could not be obtained. Most of the baseline factors were not significantly different between groups with or without anti-HBc, except for gender, age, and hepatitis B surface antibody (anti-HBs) positivity. The most common subtype of malignant lymphoma was diffuse large B-cell lymphoma (DLBCL; $\mathrm{n}=96,54.2 \%$ ). The median follow-up duration was 683 days (range, 8 to 3,147 days) from the date of ASCT. 
Table 1. Baseline characteristics according to anti-HBc status in HBsAg-negative patients prior to ASCT

\begin{tabular}{|c|c|c|c|}
\hline Characteristic & $\begin{array}{c}\text { Anti-HBc (+) } \\
\text { at ASCT }\end{array}$ & $\begin{array}{c}\text { Anti-HBc (-) } \\
\text { at ASCT }\end{array}$ & p-value \\
\hline No. of patients & $110(40.6)$ & $161(59.4)$ & \\
\hline Male/Female & $72 / 38$ & $81 / 80$ & 0.018 \\
\hline Age at ASCT (yr) & $53(16-65)$ & $48(17-65)$ & 0.001 \\
\hline Anti-HBs positive & $93(84.5)$ & $114(70.8)$ & 0.009 \\
\hline MM/Lymphoma & $38 / 72$ & $56 / 105$ & NS \\
\hline \multicolumn{4}{|l|}{ Pre-ASCT status } \\
\hline No. with elevated ALT & 5 & 8 & NS \\
\hline $\operatorname{ALT}(\mathrm{U} / \mathrm{L})$ & $19(3-59)$ & $15(3-129)$ & 0.122 \\
\hline Total bilirubin (mg/dL) & $0.4(0.2-1.1)$ & $0.4(0.2-1.9)$ & 0.323 \\
\hline Albumin $(\mathrm{g} / \mathrm{dL})$ & $4.1(2.7-5.3)$ & $4.0(1.2-5.5)$ & 0.683 \\
\hline LDH (U/L) & $247.5(107-797)$ & $266(94-2,162)$ & 0.299 \\
\hline Interval from diagnosis to ASCT (day) & $212(39-2,891)$ & $217(70-5,230)$ & 0.503 \\
\hline Cycles of CTx before ASCT & $7.0(0-36)$ & $7.0(3-43)$ & 0.477 \\
\hline MM & $4.0(0-19)$ & $5.0(3-43)$ & \\
\hline Lymphoma & $7.0(3-36)$ & $7.0(3-19)$ & \\
\hline Lines of CTx before ASCT & $1.0(0-4)$ & $2.0(1-6)$ & 0.070 \\
\hline \multicolumn{4}{|l|}{ Conditioning regimens } \\
\hline Melphalan-based in MM patients & $38(100)$ & $56(100)$ & NA \\
\hline Busulfan-based in lymphoma patients & $64(88.9)$ & $82(78.1)$ & 0.072 \\
\hline Previous rituximab therapy & $37(33.6)$ & $53(32.9)$ & NS \\
\hline Interval from last use of rituximab to ASCT (day) & $71(0-1,299)$ & $92(6-2,869)$ & 0.427 \\
\hline
\end{tabular}

Values are presented as number (\%) or median (range). Anti-HBc, hepatitis B core antibody; HBsAg, hepatitis B surface antigen; ASCT, autologous stem cell transplantation; Anti-HBs, hepatitis B surface antibody; MM, multiple myeloma; NS, not significant; ALT, alanine transaminase; LDH, lactate dehydrogenase; CTx, chemotherapy; NA, not available.

\section{Hepatitis, HBV-related hepatitis, and HBV-related death after ASCT}

Among the 271 enrolled patients, 161 were anti-HBc-negative and 110 were anti-HBc-positive at baseline (Fig. 1). Within 1 year after ASCT, 71 of the anti-HBc-negative patients and 48 of the anti-HBc-positive patients experienced hepatitis ( $44.1 \%$ vs. $43.6 \%$, p > 0.999). Neither HBsAg reversion nor HBV-related hepatitis was recorded within 1 year after ASCT, regardless of anti-HBc and anti-HBs status in both groups (Fig. 1). However, two anti-HBc-negative patients $(1.2 \%)$ and one anti-HBc-positive patient $(0.9 \%)$ developed HBV-related hepatitis at days 406, 457, and 763 after ASCT, respectively (Table 2). One of these patients died of HBV-related hepatitis. One lymphoma patient with anti$\mathrm{HBc}-$ negative showed HBsAg reversion without hepatitis at day 1,172 after ASCT. The rate of delayed HBV-related hepatitis did not differ between patients with and without anti$\mathrm{HBC}$ after adjustment for anti-HBs positivity $(\mathrm{p}=0.843$ ). Anti-HBs positivity also did not affect the rate of delayed HBV-related hepatitis after adjustment for anti-HBc status $(\mathrm{p}=0.710)$. Delayed HBV-related hepatitis occurred more frequently in MM patients (3 of 94, 3.2\%) than in lymphoma patients ( 0 of $177,0 \% ; \mathrm{p}=0.041)$.

\section{Clinical characteristics of four patients with HBsAg reversion after ASCT}

Clinical characteristics of the four patients who experienced HBsAg reversion are listed in Table 2. Patient 1 (MM, anti-HBc-positive) received $\mathrm{ASCT}$, with hematologic disease progression detected at day 98 after ASCT. HBV-related hepatitis developed at day 763 after ASCT; however, liver function improved without the need for anti-HBV therapy. Patient 2 (MM) exhibited anti-HBc positivity at the time of $\mathrm{MM}$ diagnosis; however, the patient became anti-HBc negative at the time of ASCT. Relapse of MM occurred at day 337 after ASCT, and HBV-related hepatitis developed at day 406 after ASCT, with HBV-DNA levels of 47,600 IU/mL. Entecavir $0.5 \mathrm{mg}$ daily was administered. In spite of anti-HBV therapy, however, the patient died from hepatic failure at day 453 after ASCT. Patient 3 (MM, anti-HBc-negative) 
Table 2. Clinical characteristics of four patients with HBsAg reversion after ASCT

\begin{tabular}{|c|c|c|c|c|}
\hline & Patient 1 & Patient 2 & Patient 3 & Patient 4 \\
\hline \multicolumn{5}{|l|}{ At the time of ASCT } \\
\hline Age & 60 & 54 & 54 & 46 \\
\hline Sex & Male & Male & Male & Female \\
\hline Diagnosis & MM & $\mathrm{MM}$ & $\mathrm{MM}$ & DLBCL \\
\hline Previous lines of CTx & 4 & 2 & 2 & 1 \\
\hline Prior use of rituximab & No & No & No & No \\
\hline HBV serology at diagnosis ${ }^{a}$ & $-/ \mathrm{NA} / \mathrm{NA}$ & $-1+1-$ & $-/ \mathrm{NA} / \mathrm{NA}$ & $-/ \mathrm{NA} /+$ \\
\hline HBV serology at ASCT ${ }^{\text {a) }}$ & $-1+1+$ & $-1-1-$ & $-1-1+$ & $-/-1+$ \\
\hline Anti-HBs titer (IU/L) & 11.67 & 9.76 & 44.40 & 42.65 \\
\hline HBV-DNA (IU/mL) & NA & NA & NA & NA \\
\hline $\operatorname{ALT}(\mathrm{U} / \mathrm{L})$ & 12 & 6 & 10 & 12 \\
\hline Conditioning regimen & HD Mel & VelBuMel & HD Mel & BuTT \\
\hline HBV prophylaxis & ND & ND & ND & ND \\
\hline CTx after ASCT & Steroid only & Thal, steroid, DCEP & Thal, steroid & ND \\
\hline \multicolumn{5}{|l|}{ At the time of $\mathrm{HBsAg}$ reversion } \\
\hline HBV serologya) & $+1+1-$ & $+1+1-$ & $+/ \mathrm{NA} /-$ & $+/ \mathrm{NA} /-$ \\
\hline No. of days after ASCT & 763 & 406 & 457 & 1,172 \\
\hline HBV-DNA (IU/mL) & $91,700,000$ & 47,600 & $2,200,000$ & NA \\
\hline Peak ALT (U/L) & 103 & 3,163 & 424 & 34 \\
\hline Peak total bilirubin (mg/dL) & 0.6 & 22.2 & 4.0 & 0.5 \\
\hline HBV treatment & No & Entecavir & Tenofovir & No \\
\hline Outcomes & Alive and well & Died of hepatic failure & Alive and well & Alive and well \\
\hline
\end{tabular}

HBsAg, hepatitis B surface antigen; ASCT, autologous stem cell transplantation; MM, multiple myeloma; DLBCL, diffuse large B-cell lymphoma; CTx, chemotherapy; NA, not available; Anti-HBs, hepatitis B surface antibody; HBV, hepatitis B virus; ALT, alanine transaminase; HD Mel, high-dose melphalan; VelBuMel, bortezomib, busulfan and melphalan; BuTT, busulfan and thiotepa; ND, not done; Thal, thalidomide; DCEP, dexamethasone, cyclophosphamide, etoposide and cisplatin; Anti$\mathrm{HBc}$, hepatitis B core antibody. ${ }^{\mathrm{a})} \mathrm{HBsAg} /$ anti-HBc/ anti-HBs.

developed HBV-related hepatitis at day 457 after ASCT, during maintenance therapy with thalidomide and prednisolone. Tenofovir $300 \mathrm{mg}$ daily was administered, and the patient's HBV-DNA titers became undetectable. Patient 4, who had primary DLBCL of the central nervous system (anti$\mathrm{HBc}-$ negative), did not receive any chemotherapy after ASCT. HBsAg reversion without hepatitis was incidentally detected at day 1,172 after ASCT. The status of anti-HBc at the time of diagnosis of MM or lymphoma in patients 3 and 4 could not be identified.

\section{Changes in HBV serologic markers from diagnosis to transplantation}

Among the $271 \mathrm{HBsAg-negative} \mathrm{patients} \mathrm{at} \mathrm{baseline,} 124$ were tested for anti-HBc at the time of diagnosis of $\mathrm{MM}$ or lymphoma (Fig. 2A). Among them, 55 patients (44.4\%) were anti-HBc-positive and $69(55.6 \%)$ were anti-HBc-negative. Of the 55 anti-HBc-positive patients, 50 (90.9\%) maintained their anti-HBc positivity. However, five patients (9.1\%, two lymphomas and three MMs) lost anti-HBc positivity during chemotherapy (median cycles of chemotherapy: seven cycles) and showed negativity for anti-HBc at the time of ASCT. The two lymphoma patients who lost anti-HBc positivity had received rituximab-containing chemotherapy. The disappearance rate of anti-HBc during chemotherapy was $16.7 \%$ in MM patients (median cycles of chemotherapy: four cycles) and $5.4 \%$ in lymphoma patients (median cycles of chemotherapy: seven cycles), although the difference was not statistically significant $(\mathrm{p}=0.317)$ (Table 3$)$. Rituximab-containing chemotherapy did not significantly affect the disappearance rate of anti-HBc in lymphoma patients $(\mathrm{p}=0.230)$ (Table 3).

Among the 208 patients who were HBsAg-negative at the time of ASCT and tested for anti-HBs at the time of diagnosis of MM or lymphoma, 128 (61.5\%) were anti-HBs-positive and $80(38.5 \%)$ were anti-HBs-negative at the time of diagnosis (Fig. 2B). Of the 128 anti-HBs-positive patients, 12 


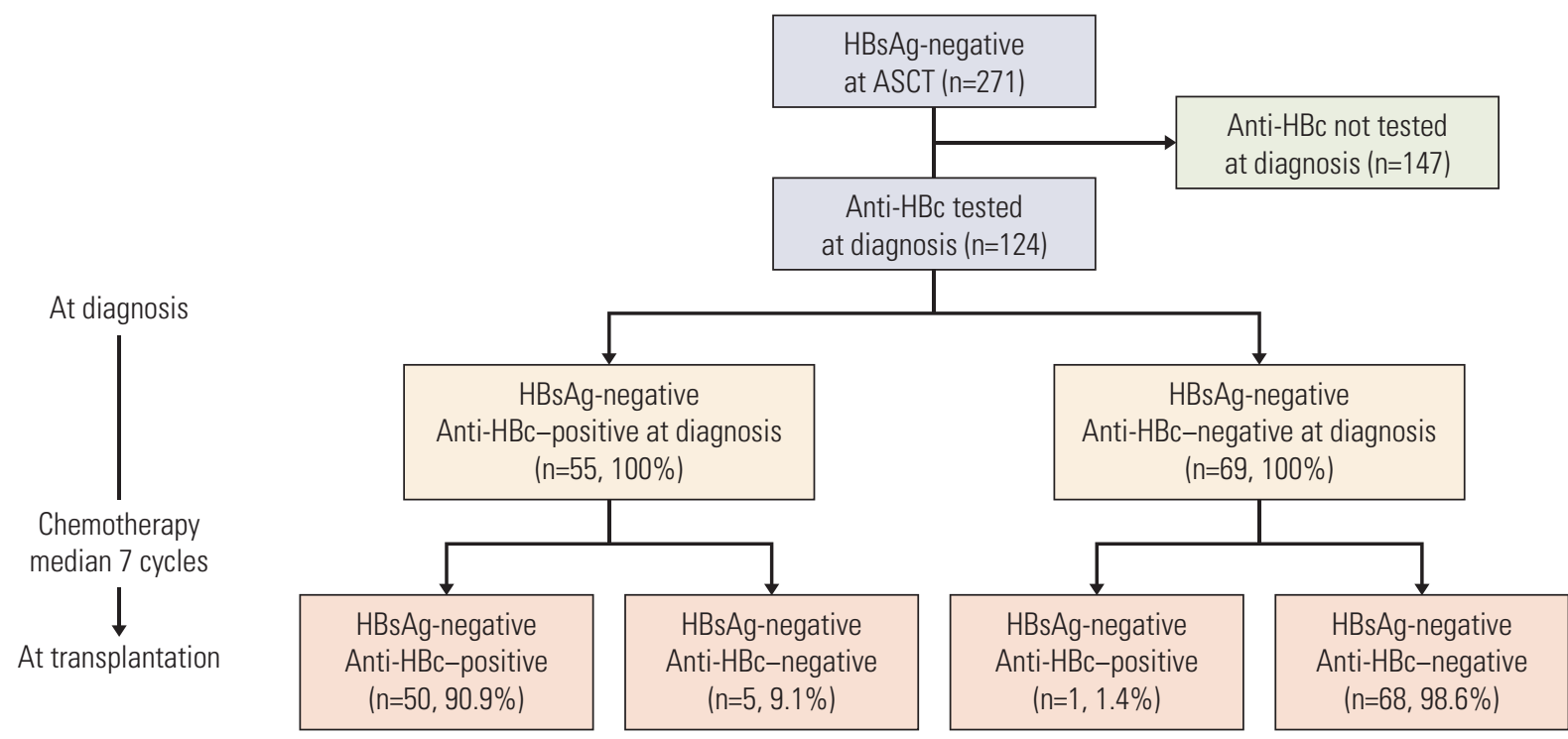

B

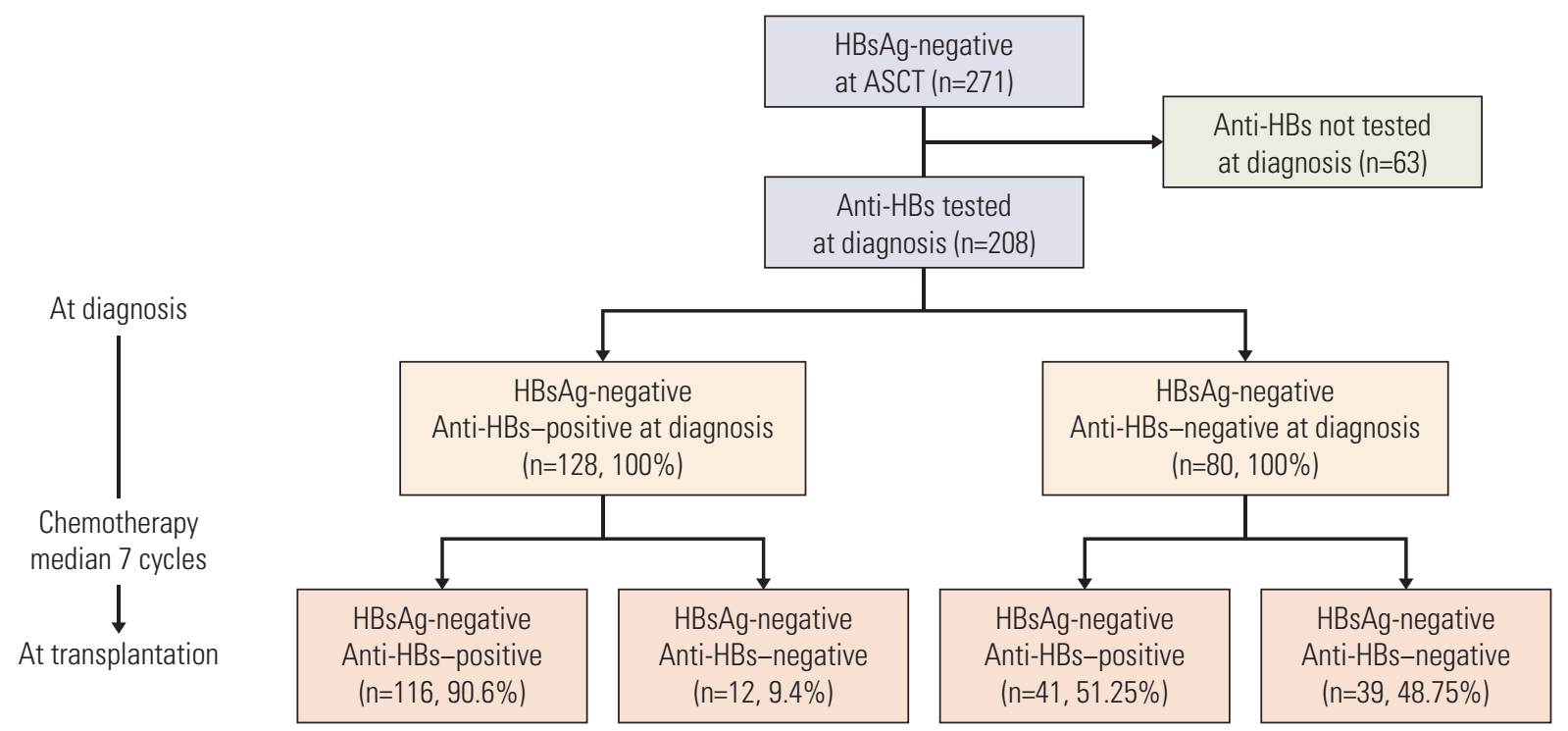

Fig. 2. Changes in hepatitis B core antibody (anti-HBc) status (A) and hepatitis B surface antibody (anti-HBs) status (B) in hepatitis B surface antigen (HBsAg)-negative patients from diagnosis to transplantation. ASCT, autologous stem cell transplantation.

(9.4\%) lost anti-HBs positivity during a median of seven cycles of chemotherapy and showed negativity for anti-HBs at the time of ASCT. The disappearance rate of anti-HBs during chemotherapy was significantly higher in MM patients (25.0\%; median cycles of chemotherapy, four cycles) than in lymphoma patients $(4.2 \%$; median cycles of chemotherapy, seven cycles) ( $p=0.002)$ (Table 3). Rituximab-containing chemotherapy did not significantly affect the rate of anti-HBs disappearance in lymphoma patients $(\mathrm{p}=0.347)$ (Table 3). 
Table 3. Disappearance of antibody during chemotherapy prior to ASCT in patients who were positive for anti-HBc or antiHBs at the time of diagnosis of MM or lymphoma

\begin{tabular}{|c|c|c|c|}
\hline Positive at diagnosis & Negative at ASCT & Positive at ASCT & p-value \\
\hline \multicolumn{4}{|l|}{ Anti-HBc } \\
\hline MM (n=18) & $3(16.7)$ & $15(83.3)$ & 0.317 \\
\hline Lymphoma $(\mathrm{n}=37)$ & $2(5.4)$ & $35(94.6)$ & \\
\hline Rituximab $^{a}(\mathrm{n}=18)$ & $2(11.1)$ & $16(88.9)$ & 0.230 \\
\hline No rituximab $\left.{ }^{\mathrm{b}}\right)(\mathrm{n}=19)$ & 0 & $19(100)$ & \\
\hline \multicolumn{4}{|l|}{ Anti-HBs } \\
\hline $\mathrm{MM}(\mathrm{n}=32)$ & $8(25.0)$ & $24(75.0)$ & 0.002 \\
\hline Lymphoma $(\mathrm{n}=96)$ & $4(4.2)$ & $92(95.8)$ & \\
\hline Rituximab $^{a}(\mathrm{n}=46)$ & $3(6.5)$ & $43(93.5)$ & 0.347 \\
\hline No rituximab ${ }^{b)}(n=50)$ & $1(2.0)$ & $49(98.0)$ & \\
\hline
\end{tabular}

Values are presented as number (\%). ASCT, autologous stem cell transplantation; Anti-HBc, hepatitis B core antibody; AntiHBs, hepatitis B surface antibody; MM, multiple myeloma. a)Patients receiving rituximab-containing chemotherapy for lymphoma before ASCT, b)Patients receiving chemotherapy without rituximab for lymphoma before ASCT.

\section{Discussion}

Anti-HBc is generally the most reliable serological marker of remote HBV infection [12]. However, in the present study, we found that $9.1 \%$ of MM and lymphoma patients experienced negative conversion of anti-HBc at the time of ASCT and remained at risk for HBV-related hepatitis. Accordingly, we suggest that $\mathrm{HBsAg}$-negative, anti-HBc-negative patients at the time of ASCT should not be considered as HBV naïve, particularly if there is not sufficient information on previous $\mathrm{HBV}$ infection. Considering that the rate of anti-HBc positivity alone is higher in areas with a high prevalence of $\mathrm{HBV}$ infection [13], the risk of HBV-related hepatitis in HBsAgnegative, anti-HBc-negative patients may theoretically be proportional to the area's prevalence of HBV infections.

$\mathrm{HBV}$-related hepatitis following $\mathrm{HBV}$ reactivation in patients with resolved $\mathrm{HBV}$ is an emerging concern during intensive chemotherapy. Several studies have evaluated the risk of developing HBV-related hepatitis in patients with HBV infection who have received intensive chemotherapy $[11,14,15]$. However, most of these previous studies have primarily focused on patients with resolved HBV infection defined as HBsAg negativity and anti-HBc positivity. Indeed, guidelines from the AASLD [3], EASL [7], and Asian-Pacific consensus statement [8] recommend that screening tests for $\mathrm{HBsAg}$ and anti-HBc in patients who are at risk of $\mathrm{HBV}$ infection should be performed before initiation of cytotoxic chemotherapy and that HBV-DNA should be monitored periodically in $\mathrm{HBsAg-negative,} \mathrm{anti-HBc-positive} \mathrm{patients.}$ Although these criteria may be useful before immunosuppressive therapy or chemotherapy, they may not be enough to determine whether patients are at risk for HBV-related hepatitis at the time of ASCT. As shown in our study, which was conducted in Korea where the prevalence of HBV infection and anti-HBc positivity alone is high [16], a considerable number of patients with MM or lymphoma lost anti-HBc positivity during chemotherapy; moreover, the rate of $\mathrm{HBV}$ related hepatitis did not differ between patients who were anti-HBc-negative and those who were anti-HBc-positive prior to undergoing ASCT. Our finding of a relatively low rate of $\mathrm{HBV}$-related hepatitis in anti-HBc-positive patients may be due in part to the relatively short follow-up duration of our study (median, 683 days) because a recent study suggested that most HBV reactivations occur more than 19 months after transplantation [17]. Nevertheless, our data suggest that the risk of HBV-related hepatitis in HBsAg-negative, anti-HBc-negative patients at the time of ASCT is not negligible, particularly in HBV endemic areas, such as SouthEast Asia and Western Pacific Regions [18].

Notably, the absolute percentage of patients who lost anti$\mathrm{HBc}$ during chemotherapy prior to ASCT was higher in MM patients $(16.7 \%)$ than in lymphoma patients (5.4\%). However, the difference was not statistically significance. Given that the number of patients who were analyzed to determine the disappearance rate of anti-HBc during chemotherapy was small (18 MM and 37 lymphoma), we could not conclude whether or not MM patients experience a higher disappearance rate of anti-HBc during chemotherapy than lymphoma patients. Nevertheless, since MM is an incurable disease by nature of dysfunctional humoral immunity, we suspect that $\mathrm{MM}$ patients are at a high risk of anti-HBc disappearance. Meanwhile, our study also found that reversion of anti-HBs occurs more frequently in MM patients than in lymphoma 
patients during chemotherapy. In addition, HBV-related hepatitis developed more frequently in MM patients. Similar to these findings, both loss of anti-HBs and MM were reported as independent risk factors for HBV reactivation previously $[19,20]$. Therefore, we recommend that MM HBsAg-negative, anti-HBc-negative patients should be carefully evaluated for the risk of HBV-related hepatitis during chemotherapy.

Rituximab is a chimeric anti-CD20 monoclonal antibody that is extensively used in various B-cell lymphoid malignancies and immune-mediated diseases [21]. Although rituximab-based treatments are known to reduce the levels of circulating antibodies [22], we did not find significantly increased disappearance rates of anti-HBc or anti-HBs in lymphoma patients who received a rituximab-containing chemotherapy as induction chemotherapy. In addition, none of those receiving rituximab-containing induction chemotherapy showed HBV-related hepatitis after ASCT. However, the small number of patients limited our ability to reach a definitive conclusion.

This study has a few limitations. First, HBV serology was tested in accordance with clinical suspicions, which could introduce potential bias. Second, as HBV-DNA levels were not tested regularly, we could not identify the appearance of HBV-DNA in the serum (e.g., HBV reactivation) in the absence of HBsAg. Therefore, the rate of HBV reactivation may have consequently been underestimated, especially in patients who had treated with rituximab which is one of the major risk factors of HBV reactivation [11]. Because it is not necessary to conduct anti-HBV treatments in patients who have only positive findings of HBV-DNA without elevation of biochemical markers for hepatitis, the determination of the patients with HBV-related hepatitis may be useful in clinical practice. Third, vaccination history could not be obtained.
Considering that universal vaccination of all infants has been performed since 1991 in Korea [23], the possibility of aggressive vaccination, which may reduce the rate of $\mathrm{HBV}$-related hepatitis, could not be excluded. Finally, due to the retrospective nature of this study, only a small number of patients had results on anti-HBc or anti-HBs at the time of diagnosis of MM or lymphoma. As only one of three patients who were anti-HBc-negative at baseline and experienced HBsAg reversion after ASCT was identified to have anti-HBc at the time of diagnosis, our study had insufficient data with which to reveal an association between disappearance of anti-HBc and HBV-related hepatitis. Despite these limitations, our study demonstrates for the first time the potential risk of anti-HBc disappearance and HBV-related hepatitis in MM and lymphoma patients. Larger, prospective studies are needed to confirm the results of our study.

In summary, our study showed that $16.7 \%$ of MM patients and $5.4 \%$ of lymphoma patients who were HBsAg-negative and anti-HBc-positive at the time of diagnosis of MM or lymphoma had lost their anti-HBc positivity during chemotherapy prior to ASCT and remained at risk of HBV-related hepatitis. Hence, patients who are HBsAg-negative and anti$\mathrm{HBc}-$ negative at the time of ASCT should also be considered at risk of HBV-related hepatitis, particularly MM patients in $\mathrm{HBV}$ endemic areas. Not only screening tests for HBV serologic markers and HBV-DNA levels at the time of ASCT but also a comprehensive review of previous HBV history, including serologic markers and HBV-DNA, may necessary to determine which patients are at risk of HBV-related hepatitis after ASCT.

\section{Conflicts of Interest}

Conflict of interest relevant to this article was not reported.

\section{References}

1. Pei SN, Chen CH. Risk and prophylaxis strategy of hepatitis $\mathrm{B}$ virus reactivation in patients with lymphoma undergoing chemotherapy with or without rituximab. Leuk Lymphoma. 2015;56:1611-8.

2. Hwang JP, Lok AS. Management of patients with hepatitis B who require immunosuppressive therapy. Nat Rev Gastroenterol Hepatol. 2014;11:209-19.

3. Lok AS, McMahon BJ. Chronic hepatitis B: update 2009. Hepatology. 2009;50:661-2.

4. Gu HR, Shin DY, Choi HS, Moon CH, Park SC, Kang HJ. HBV reactivation in a HBsAg-negative patient with multiple myeloma treated with prednisolone maintenance therapy after autologous HSCT. Blood Res. 2015;50:51-3.

5. Yoshida T, Kusumoto S, Inagaki A, Mori F, Ito A, Ri M, et al. Reactivation of hepatitis B virus in HBsAg-negative patients with multiple myeloma: two case reports. Int J Hematol. 2010;91:844-9.

6. Yeo W, Chan PK, Zhong S, Ho WM, Steinberg JL, Tam JS, et al. Frequency of hepatitis B virus reactivation in cancer patients undergoing cytotoxic chemotherapy: a prospective study of 626 patients with identification of risk factors. J Med Virol. 2000;62:299-307.

7. European Association for the Study of the Liver. EASL clinical practice guidelines: management of chronic hepatitis B virus 
infection. J Hepatol. 2012;57:167-85.

8. Liaw YF, Kao JH, Piratvisuth T, Chan HL, Chien RN, Liu CJ, et al. Asian-Pacific consensus statement on the management of chronic hepatitis B: a 2012 update. Hepatol Int. 2012;6:53161.

9. Ceneli O, Ozkurt ZN, Acar K, Rota S, Aki SZ, Yegin ZA, et al. Hepatitis B-related events in autologous hematopoietic stem cell transplantation recipients. World J Gastroenterol. 2010;16: 1765-71.

10. Guillaume T, Rubinstein DB, Symann M. Immune reconstitution and immunotherapy after autologous hematopoietic stem cell transplantation. Blood. 1998;92:1471-90.

11. Yeo W, Chan TC, Leung NW, Lam WY, Mo FK, Chu MT, et al. Hepatitis $B$ virus reactivation in lymphoma patients with prior resolved hepatitis B undergoing anticancer therapy with or without rituximab. J Clin Oncol. 2009;27:605-11.

12. Laperche S, Guitton C, Smilovici W, Courouce AM. Blood donors infected with the hepatitis B virus but persistently lacking antibodies to the hepatitis B core antigen. Vox Sang. 2001;80:90-4.

13. Ponde RA, Cardoso DD, Ferro MO. The underlying mechanisms for the 'anti-HBc alone' serological profile. Arch Virol. 2010;155:149-58.

14. Lu S, Xu Y, Mu Q, Cao L, Chen J, Zhu Z, et al. The risk of hepatitis $B$ virus reactivation and the role of antiviral prophylaxis in hepatitis B surface antigen negative/hepatitis B core antibody positive patients with diffuse large B-cell lymphoma receiving rituximab-based chemotherapy. Leuk Lymphoma. 2015;56:1027-32.

15. Hsu C, Tsou HH, Lin SJ, Wang MC, Yao M, Hwang WL, et al. Chemotherapy-induced hepatitis $B$ reactivation in lymphoma patients with resolved HBV infection: a prospective study. Hepatology. 2014;59:2092-100.

16. Kang SY, Kim MH, Lee WI. The prevalence of "anti-HBc alone" and HBV DNA detection among anti-HBc alone in Korea. J Med Virol. 2010;82:1508-14.

17. Yoo JJ, Cho EJ, Cho YY, Lee M, Lee DH, Cho Y, et al. Efficacy of antiviral prophylaxis in $\mathrm{HBs} \mathrm{Ag}$-negative, anti-HBc positive patients undergoing hematopoietic stem cell transplantation. Liver Int. 2015;35:2530-6.

18. Papastergiou V, Lombardi R, MacDonald D, Tsochatzis EA. Global epidemiology of hepatitis B virus (HBV) infection. Curr Hepatol Rep. 2015;14:171-8.

19. Han JW, Yang H, Lee HL, Bae SH, Choi JY, Lee JW, et al. Risk factors and outcomes of hepatitis $B$ virus reactivation in hepatitis B surface antigen negative patients with hematological malignancies. Hepatol Res. 2016;46:657-68.

20. Uhm JE, Kim K, Lim TK, Park BB, Park S, Hong YS, et al. Changes in serologic markers of hepatitis $B$ following autologous hematopoietic stem cell transplantation. Biol Blood Marrow Transplant. 2007;13:463-8.

21. Seto WK, Chan TS, Hwang YY, Wong DK, Fung J, Liu KS, et al. Hepatitis $B$ reactivation in patients with previous hepatitis $B$ virus exposure undergoing rituximab-containing chemotherapy for lymphoma: a prospective study. J Clin Oncol. 2014;32:3736-43.

22. Liossis SN, Sfikakis PP. Rituximab-induced B cell depletion in autoimmune diseases: potential effects on $\mathrm{T}$ cells. Clin Immunol. 2008;127:280-5.

23. Kim H, Shin AR, Chung HH, Kim MK, Lee JS, Shim JJ, et al. Recent trends in hepatitis B virus infection in the general Korean population. Korean J Intern Med. 2013;28:413-9. 